\title{
Capital Psicológico: un constructo fundacional dentro de la psicología organizacional positiva
}

\author{
Psychological Capital: a foundational construct in positive organizational psychology
}

Andrés Cabezas Corcione ${ }^{1}$

\section{RESUMEN}

El presente artículo propone dar a conocer la importancia del capital psicológico como constructo fundacional, dentro de la psicología organizacional positiva, sosteniendo y evidenciando su impacto positivo tanto en el desempeño laboral, como en la felicidad y satisfacción vital de los trabajadores. Para esto en primera instancia se revisará brevemente, qué se entiende por psicología organizacional positiva, para luego poder realizar una definición conceptual del CaPsi y sus cuatro dimensiones; esperanza, resiliencia, optimismo y autoeficacia. Concluyendo que este constructo cumple con los cinco criterios de inclusión propuestos por Youssef, Luthans \& Avolio en el año 2007, lo cual permite el estudio y aplicación de las capacidades y fortalezas que pueden ser medidas y desarrolladas para mejorar el rendimiento (Nelson y Cooper, 2007), se destaca que pese a los pocos estudios que se han realizado hasta el momento, los resultados han evidenciado relaciones altamente significativas entre el capital psicológico y el desempeño laboral, aclarando que los instrumentos utilizados en cada investigación, han demostrado alta validez y confiabilidad.

Palabras clave: Psicología Organizacional Positiva, Capital Psicológico, Esperanza, Optimismo, Resiliencia, Autoeficacia

\begin{abstract}
The present article aims to raise awareness of the importance of the psychological capital as a fundamental construct within the positive organizational psychology. It maintains and demonstrates its positive impact on job performance, as well as happiness and life satisfaction of workers. For this it will be briefly reviewed what is meant by positive organizational psychology, so that a conceptual definition of the CaPsi and its four dimensions; hope, resilience, optimism and self-efficacy, can be performed. It concludes that this construct meets the five criteria of inclusion, proposed by Youssef, Luthans \& Avolio in the year 2007, which allows the study and application of the skills and strengths that can be measured and developed to improve performance (Nelson and Cooper, 2007). The article emphasizes that despite the few studies that have been conducted so far, the results showed highly significant relationships between psychological capital and job performance, and clarifies that the instruments used in each investigation, have shown high validity and reliability.
\end{abstract}

Keywords: Positive Organizational Psychology, Psychological Capital, Hope, Optimism, Resilience, Self-efficacy

${ }^{1}$ PhD, Director del Centro Latinoamericano de Psicología Positiva Aplicada-CELAPPA. Profesor de la Facultad de Ciencias Sociales y Humanidades de la Universidad Autónoma de Chile. 


\section{INTRODUCCIÓN}

Uno de los aspectos relevantes a considerar dentro del capital psicológico, antes de definir conceptualmente este constructo fundacional de la POP, es la propuesta de los cinco criterios de inclusión postulados por Youssef, Luthans y Avolio (2007), los cuales surgen con el fin de evitar la proliferación de constructos emergentes, en tanto se sostendrá al capital psicológico como un constructo basado en la teoría e investigación, el cual puede ser medido a través de instrumentos válidos y confiables, siendo casi único en su ámbito, generando un impacto positivo en el desempeño y satisfacción de los trabajadores. Para esto será necesario presentar una definición de psicología organizacional positiva y luego el de capital psicológico, el cual está compuesto por; esperanza, optimismo, resiliencia y autoeficacia, siendo cada uno de estos componentes presentados desde investigaciones recientes. (Youssef, Luthans y Avolio). Dentro de la psicología organizacional positiva existen constructos fundacionales y emergentes, siendo el capital psicológico o CaPsi considerado como fundacional. Este concepto surge dentro de la teorización realizada por Luthans et. al (2004), como un nuevo concepto distinto al de capital humano y social, el cual está integrado por cuatro componentes-optimismo, esperanza, resiliencia y autoeficacia- los cuales ya habían sido estudiados previamente por autores pioneros ( Snyder, 2000; Parker, 1998; Seligman,1991) en la psicología positiva, presentándose de modo imperante antes de definir el Capsi, comprender qué se entiende por psicología organizacional positiva junto con conocer brevemente el contexto en el cual surge.

\section{Comportamiento Organizacional Positivo desde la psicología positiva}

Durante muchos años el objeto de estudio de la psicología se ha dirigido al sufrimiento humano, comprendiéndolo como desorden, déficit o trastorno, el cual ha sido conceptualizado por distintas corrientes y escuelas psicológicas, las que se han desarrollado principalmente en tres campos de aplicación psicología clínica, educacional y laboral - los que buscan la resolución de problemas, comprensión y explicación de fenómenos y principalmente reducir la disonancia que existe en el ser humano al experimentar un estado psicológico negativo, con lo cual frente a esto Csikszentmihalyi (1998), sostiene que la psicología no debería ocuparse tanto de reparar el daño psicológico y remitir los síntomas, sino que debería centrar su objeto de estudio en cómo se potencian las cualidades positivas, que cada ser humano posee.
La psicología organizacional como campo de aplicación y área de estudio ha centrado su énfasis en reconocer los conflictos que surgen dentro de las organizaciones y sus posibles mecanismos resolutivos, propiciando un clima laboral idóneo para el desarrollo organizacional, el que permita mejorar el desempeño de los trabajadores, sin embargo la Psicología Organizacional Positiva surge como un nuevo enfoque en la gestión de los recursos, basándose en la teoría e investigación de la psicología positiva (Peterson \& Seligman, 2004; Seligman \& Csikszentmihalyi, 2000; Sheldon \& King , 2001; Zinder \& Lopez, 2002), vista como una psicología llevada al lugar de trabajo.

El término Comportamiento Organizacional Positivo es acuñado por Luthans en el año 2002 siendo definido como "el estudio y la aplicación de las capacidades psicológicas y las fortalezas que pueden ser medidas, desarrolladas y gestionadas eficazmente para mejorar el rendimiento en los lugares de trabajo hoy en día” (p.59), para este autor no sólo basta con conceptualizar un fenómeno desde un nuevo punto de análisis, sosteniendo que para evitar la proliferación indiscriminada de constructos se debe establecer un conjunto de criterios que cada uno de ellos debe cumplir, estableciéndose cinco criterios de inclusión, los cuales deben estar basados en la teoría, ser medidos a través de instrumentos válidos y confiables, ser relativamente únicos en el campo del comportamiento organizacional, tener un impacto positivo sobre la satisfacción y el rendimiento laboral y más que rasgos fijos, ser estados susceptibles de cambio y desarrollo (Omar, 2010).

\section{Capital Psicológico: Una definición conceptual}

Como se mencionaba anteriormente cada constructo generalmente se compone de distintos conceptos que al momento de cumplir con los criterios de inclusión, dan la posibilidad de ser operacionalizados y por lo tanto medidos, es el caso del capital psicológico, introducido por Luthans \& Luthans (2004) en su estudio titulado Positive psychological capital: Beyond human and social capital el cual contempla como objetivo establecer una medida comparable y complementaria al capital humano, físico y social, sosteniendo que es un concepto multidimensional que se integra a partir de la conjunción entre los conceptos esperanza, resiliencia, optimismo y autoeficacia, en tanto como dice Omar (2010)

Este constructo multidimensional ha sido definido como "el estado psicológico positivo caracterizado por: 1) tener confianza (autoeficacia) para asumir y 
llevar a cabo con éxito una tarea desafiante; ") hacer una atribución positiva (optimismo) sobre el éxito actual y futuro; 3) perseverar en la búsqueda de los objetivos $y$, en caso necesario, redireccionar los esfuerzos hacia las metas (esperanza) a fin de tener éxito, y 4) a pesar de estar acosado por los problemas, fortalecerse en la adversidad (resiliencia) para alcanzar el éxito” (p.139)

Por lo tanto el capital psicológico o PsyCap es concebido como la valoración positiva de las circunstancias y posibilidades de éxito basadas en el esfuerzo y perseverancia (Luthans \& Youssef, 2007), el que es extendido hacia las organizaciones como un constructo emanado desde la psicología positiva, intentando contribuir a las ventajas competitivas de las organizaciones (Page \& Donohue, 2004), puesto que desde la visión basada en los recursos en la empresa; el uso óptimo del capital humano puede ser clave en la ventaja competitiva entre organizaciones, al ser muy difícil de replicar (Barney, 1991).

Este constructo fundacional, también es considerado como emergente por algunos autores (Luthans \& Youssef, 2004; Luthans, Avey, Patera, 2008; Combs, Luthans \& Griffith, 2009, Cetin, 2011) puesto que sigue siendo estudiado con el fin de ser medido a través de instrumentos válidos y confiables en su operacionalización, en tanto existen otros constructos que se encuentran en el mismo camino, como lo son el de capital social y el de capital humano.

Es importante considerar que debe existir una distinción entre los conceptos que constituyen este constructo-autoeficacia, optimismo, resiliencia y esperanza-, puesto que no siempre existirá claridad conceptual, tomando como ejemplo que la esperanza fácilmente puede ser confundida con el optimismo (Page \& Donohue, 2004) y como dice Osigweh (1989) el desarrollo de definiciones claras de ciertos conceptos es muy importante para la mejora de la construcción e investigación organizacional.

\section{Dimensiones del Capital Psicológico}

El CapPsi fue analizado por Luthans y Avolio (2006), con el fin de comprobar si este constructo cumplía con uno de los criterios de inclusión- más que rasgos fijos ser estados susceptibles de cambio y desarrollo- concluyendo que no sería tan estable, estando más abierto hacia los conceptos rasgos; comprendiendo que estados y rasgos son considerados como categorías independientes y dicotómicas de cada constructo, por lo tanto el CapPsi sería maleable, modificable y por ende susceptible a un cambio o desarrollo (Omar, 2010). Esto se realizó gracias a los estudios previos sobre esperanza, resiliencia, optimismo y autoeficacia realizados por distintos autores (Bandura, 2008, Zinder, 2005; Carver y Shaier, 2005; Seligman, 2001; Masten y Reed, 2002).

\section{El rol de la esperanza}

La dimensión esperanza dentro de la psicología positiva posee un desarrollo y soporte teórico y empírico considerable (Luthans, Avey, Avolio \& Peterson, 2010), asociado a la teoría propuesta por Snyder, Irving y Anderson (1991), como un estado motivacional positivo sustentado en un sentido de éxito el cual deriva interactivamente de la voluntad de alcanzar ciertas metas y la consideración de los caminos a seguir para lograrlas, en tanto Page y Donohue (2004) sostienen que este constructo se divide en 2 dimensiones; la motivación-willpower- y las vías o caminos -pathways-, en tanto el componente motivacional se compone de las expectativas para alcanzar un determinado objetivo, o la intención o voluntad para lograr un efecto deseado y las vías o caminos vendrían a complementar la motivación ofreciendo vías alternativas para conseguir los objetivos vistos como recursos psicológicos, aclarando que como afirma Snyder et al. (1996), será necesario que estos dos componentes se encuentren juntos puesto que no basta sólo con uno para conseguir una meta u objetivo, siendo totalmente complementarios, a lo que Omar agrega

Los sujetos que tienen elevada esperanza utilizan la planificación contingente en previsión de obstáculos en la consecución de objetivos o subobjetivos, e identifican de manera proactiva múltiples rutas para alcanzarlos. En otras palabras, la esperanza puede ser definida como la voluntad de triunfar y la capacidad para identificar y seguir el camino al éxito (Omar, 2010, p.140).

Los estudios realizados sobre la esperanza han demostrado que este constructo posee una gran validez al momento de establecer correlaciones ya sea en el área clínica, educacional o deportiva, no obstante pocos son los estudios realizados con esta dimensión en el lugar de trabajo, en tanto el primer estudio realizado en esta línea de investigación fue realizado por Luthans, Avolio, Walumbwa y Li (2005), en el cual se estudió el capital psicológico de los trabajadores chinos, explorando su relación con el desempeño. También se estudió la relación entre el CaPsi, desempeño retención y satisfacción, encontrando correlaciones positivas (Peterson y Luthans, 2003). Dentro de estos estudios se utilizó el Psychological Capital Questionnaire (ver Luthans et al. 2007) el cual según Luthans, Avey Clapp- 
Smith y Weixing Li (2008) ha presentado una gran confiabilidad $(\alpha=68)$ en distintas aplicaciones $\mathrm{y}$ en distintas traducciones, afirmando que las cuatro dimensiones que lo componen -esperanza, optimismo, autoeficacia y resiliencia- cumplen perfectamente con los criterios de inclusión, al estar basados en la teoría e investigación, siendo correctamente operacionalizados en su primera etapa y validados en una segunda etapa.

\section{EI rol de la Resiliencia}

La resiliencia tiene un gran correlato teórico e investigativo en la psicología clínica y positiva, basado en los estudios de niños y adolescentes que lograron superar grandes adversidades (Luthans et al 2009), siendo definida como la capacidad de hacer frente a la adversidad o a las situaciones estresantes (Masten, Best \& Garmezy; Masten 2001; Luthans, 2001) y aplicada al ámbito laboral, sería "la capacidad psicológica de recuperarse positivamente de la adversidad, de la incertidumbre, del conflicto y del fracaso" (Omar, 2010, p. 141). En tanto, este componente es reconocido como una capacidad que todos los individuos poseen, existiendo la posibilidad de desarrollarlo (Masten, 2001), lo cual ha sido estudiado en distintos ámbitos psicológicospsicología clínica, educacional, social y comunitaria-, no obstante en el ámbito laboral no es mucho lo que se ha investigado, siendo Luthans et al (2005) quien en su estudio realizado con trabajadores chinos logró encontrar una relación significativa entre la resiliencia y el nivel de aceptación a los cambios evidenciándose el impacto que tiene este componente en cuanto a la flexibilidad y apertura al cambio en el entorno laboral. Por otro lado como sostiene Omar (2010), existe un estudio realizado en el año 1987 por Maddi el cual concluyó que los trabajadores resilientes de una organización, mantenían su felicidad, salud y rendimiento aún en periodos de reducción masiva de personal. Finalmente, la resiliencia genera un claro impacto en el desempeño laboral pudiendo ser desarrollada en un nivel individual (Luthans \& Youssef, 2004).

\section{El rol del optimismo}

Dentro de la psicología positiva, el optimismo utiliza un lugar relevante al ser estudiado por Seligman (1991) en su teoría atribucional, definiendo al sujeto optimista como alguien que realiza atribuciones internas, globales y estables frente a acontecimientos positivos y atribuciones externas, específicas e inestables frente a los eventos negativos. Para la psicología organizacional positiva el optimismo es asociado con la atribución interna y positiva frente a acontecimientos que incluyen motivación y emociones positivas (Luthans, 2002; Luthans et al., 2007; Schneider, 2001); por lo tanto, es un proceso controlado que cuenta con una evaluación realista de las situaciones de desempeño.

Al igual que los demás componentes del CaPsi, el optimismo es considerado como una dimensión emergente en el lugar de trabajo al no contar con muchos estudios, en tanto Seligman (1998) encontró una relación significativa entre el optimismo y el desempeño en agentes de ventas, como así también Luthans et al. (2005) encontró una relación significativa entre optimismo y desempeño en trabajadores chinos, el cual unos años más adelante fue confirmado por Youssef \& Luthans (2007), al sostener que el optimismo se relacionaba no tan sólo con el desempeño sino también con la felicidad y satisfacción vital.

\section{El rol de Autoeficacia}

Esta dimensión es acuñada en la teoría sociocognitiva de Bandura en el año 1986 siendo definida por Stajkovic \& Luthans (1998), como la creencia positiva y autoconfianza en las propias habilidades para movilizar la motivación, recursos cognitivos y cursos de acción necesarios para conseguir un objetivo en un determinado contexto. A diferencia de los demás componentes del CaPsi, la autoeficacia posee un amplio campo de investigación en el lugar de trabajo, encontrándose relaciones altamente significativas entre este componente y el desempeño laboral, inclusive en estudios realizados en culturas altamente colectivas como lo es caso de China, se logró observar que los sujetos que logran desarrollar esta habilidad consiguen un mejor rendimiento, desempeño y satisfacción en sus resultados.

\section{CONCLUSIONES}

Dentro de la psicología organizacional positiva, existen distintos constructos los cuales buscan contribuir al desempeño, calidad de vida y felicidad de los sujetos en su lugar de trabajo, siendo el CaPsi considerado como fundacional y por el contrario como emergente por la mayoría de los autores, revistiendo gran relevancia teórica, metodológica y práctica, al desplazar sustancialmente al capital humano y social en los recursos humanos, en cuanto a la relación significativa evidenciada respecto a las variables; desempeño, felicidad, satisfacción y confianza en el lugar de trabajo. Siendo importante destacar que cada dimensión que lo compone logra cumplir con los criterios de inclusión propuestos por 
Luthans, Youssef y Avolio (2007); al estar basados en la teoría e investigación, ser medidos a través de instrumentos válidos y confiables-positive capital questionnaire-, ser relativamente únicos en el campo del comportamiento organizacional y teniendo un impacto positivo en la satisfacción laboral y el rendimiento laboral.

Finalmente, comprendiendo que el CaPsi es la valoraciónpositivadelascircunstanciasyposibilidades de éxito basada en el esfuerzo y perseverancia, junto con sostener que al ser un estado y no un rasgo, puede ser desarrollado, modificado y aprendido. Planteando de forma imperante, la necesidad de realizar en próximos estudios la inclusión de este constructo en la psicología organizacional positiva.

\section{Declaración de financiamiento y de conflicto de intereses:}

El estudio fue financiado por los autores, quienes declaran no tener algún tipo de conflicto de interés en la investigación realizada.

\section{Correspondencia:}

\section{Andrés Cabezas Corcione}

Centro Latinoamericano de Psicología Positiva Aplicada.

Facultad de Ciencias Sociales y Humanidades, Universidad Autónoma de Chile. Dirección 5 poniente 1670 .

e-mail: andrescabezas@acpp.cl

\section{REFERENCIAS BIBLIOGRÁFICAS}

Bandura, A. (2008). An agentic perspective on positive psychology. In S. J. Lopez (Ed.), Positive psychology: Exploring the best in people, 1, 167-196.

Barney, J. (1991). Firm Resources and Sustained Competitive Advantage. Journal of Management March 17, 99120. En Page, L. F., \& Donohue, R. (2004). Positive psychological capital: A preliminary exploration of the construct. Paper Series, Monash University, 51(4) 1-10

Combs, G. M. (2006). Psychological capital development: Toward a micro-intervention. Journal of Organizational Behavior, 27, 387-393.

Csikszentmihalyi, M. (1998). Fluir. Una psicología de la felicidad, Barcelona, Kairós.

Lin, N., Cook, K., \& Burt, R. (2001). Social Capital: Theory and Research. NY;

Luthans, F \& Youssef, C. M. (2007). Emerging positive organizational behavior. Journal or Management, 3, 321-349

Luthans, F. (2002a). Positive organizational behavior: developing and maintaining psychological strengths. Academy of Management Executive, 16, 57-72.

Luthans, F. (2002b). The need for and meaning of positive organizational behavior. Journal of Organizacional Behavior, 23, 695-706.

Luthans, F. (2003). Positive organizational behavior: implications for leadership and HR development and motivation. En Porter ,L, Bigley, G, y Steers, R, M (Eds.), Motivation and work behavior p. 178-195 New York: McGraw-Hill/Irwin.

Luthans, F., Avey, J. B., \& Patera, J. (in press) Experimental analysis of a web-based training intervention to develop positive psychological capital. Academy of Management Learning and Education Journal.

Luthans, F., Avey, J. B., \& Patera, J. L. (2008). Experimental analysis of a web-based training intervention to develop positive psychological capital. Academy of Management Learning and Education, 7, 209 -221.

Luthans, F., Avolio, B. J., Walumbwa, F. O., \& Li, W. (2005). The psychological capital of Chinese workers: Exploring the relationship with performance. Management and Organization Review, 1, 249 -271.

Luthans, F., Luthans, K.W., \& Luthans, B. C. (2004). Positive psychological capital: Beyond human and social capital. Business Horizons, 41(1), 45-50

Luthans, F., Norman, S.M., Avolio, B.J., \& Avey, J.B. (2008). The mediating role of psychological capital in the supportive organizational climate-employee performance relationship. Journal of Organizational Behavior, 29, 219-238

Masten, A. S. (2001). Ordinary magic: Resilience processes in development. American Psychologist, 56, 227-239.

Masten, A. S., \& Reed, M. G. J. (2002). Resilience in development. In C. R. Snyder, \& S. López (Eds.), Handbook of positive psychology (pp. 74-88). Oxford, UK.: Oxford University Press. En Luthans, F., Norman, S.M.,Avolio, B.J., \& Avey, J.B. (2008). The mediating role of psychological capital in the supportive organizational climate-employee performance relationship. Journal of Organizational Behavior, 29, 219-238

Omar, A. (2010). Las organizaciones positivas. En Castro Solano, A (comp). (2010). Fundamentos de la psicología positiva. Buenos Aires: Paidós.

Osigweh, C.A. B. (1989). Concept fallibility in organizational science. The Academy of Management Review, 14(4), 579-584

Page, L. F., \& Donohue, R. (2004). Positive psychological capital: A preliminary exploration of the construct. Paper Series, 51(4) 1-10

Peterson, S. Y Seligman, M.E.P. (2004). Carácter strenghts 
before and alter September 11. Psychological Science, $14,381-384$

Peterson, S., \& Luthans, F. (2003). The positive impact of development of hopeful leaders. Leadership and Organization Development Journal, 24, 26-31.

Omar, A. (2010). Las organizaciones positivas. En Castro Solano, A (comp). (2010). Fundamentos de la psicología positiva. Buenos Aires: Paidós.

Scheier, M. F., \& Carver, C. S. (1985). Optimism, coping, and health: Assessment and implications of generalizad outcome expectancies. Health Psychology, 4, 219-247.

Seligman, M \& Csikszentmihalyi, M. (2000). Positive psychology: An introduction. American Psychologist, 55, 5-14

Sheldon, K., M \& King, L. (2001). Why positive psychology is necessary. American Psychologist, 56(3), 216-217

Snyder, C. R, and Lopez, S.J. (eds) (2002). Handbook of Positive Psychology, Oxford, UK: Oxford University Press. En Luthans, F, Avey, J.B, Clapp-Smith, R. \& Weixing Li. (2008). More evidence on the value of Chinese workers` psychological capital: A potencially unlimited competitive resorce?. The International Journal of Human Resource Management, 19(5), 818-827

Snyder, C. R., Irving, L. M., \& Anderson, J. R. (1991). Hope and health. Luthans, F., Norman, S.M., Avolio, B.J., \&
Avey, J.B. (2008). The mediating role of psychological capital in the supportive organizational climate-employee performance relationship. Journal of Organizational Behavior, 29, 219-238

Snyder, C. R., Sympson, S., Ybasco, F., Borders, T., Babyak, M., \& Higgins, R. (1996). Development and validation of the state hope scale. Journal of Personality and Social Psychology, 70, 321-335

Stajkovic, A., \& Luthans, F. (1998). Self-efficacy and workrelated performance: A meta-analysis. Psychological Bulletin, 44, 580-590.

Stewart, T. A, (2001). The wealth of knowledge: Intellectual capital and the twenty -first centuryorganization. New York. En Page, L. F., \& Donohue, R. (2004). Positive psychological capital: A preliminary exploration of the construct. Paper Series, 51(4) 1-10

Snyder, C.R. (2000). Handbook of Hope. San Diego, CA: Academic Press

Parker, S. (1998). Enhancing role-breath self efficacy: The roles of job enrichment and other organizational interventions. Journal of Applied Psychology, 83, 835852. http://dx.doi.org/10.1037/0021-9010.83.6.835

Seligman, MEP (1991). Learned Optimism. New York: Kpnof

Recibido: 17/09/2015

Aceptado: 11/11/2015 